\title{
DOSES DE ADUBAÇÃO NITROGENADA E POTÁSSICA EM COBERTURA Na CULTURA DA CANA-DE-AÇÚCAR, PRIMEIRASOCA, COM E SEM IRRIGAÇÃO
}

\author{
Levels of manuring in the sugar-cane crop, first leaf, with and without irrigation
}

\author{
Maria Vilian Pereira da Silva Moura ${ }^{1}$, Carlos Henrique de Azevedo Farias ${ }^{2}$, Carlos Alberto Vieira de Azevedo \\ José Dantas Neto ${ }^{3}$, Hamilton Medeiros de Azevedo ${ }^{3}$, Roberto Vieira Pordeus ${ }^{4}$
}

\begin{abstract}
RESUMO
$\mathrm{O}$ presente trabalho teve como objetivo avaliar os efeitos de diferentes doses de $\mathrm{N}$ e $\mathrm{K}_{2} \mathrm{O}$, aplicados em cobertura, no desenvolvimento e rendimento de colmos, açúcar e álcool da cana-soca (Saccharum spp, cultivar SP79 1011) cultivada com e sem irrigação, em área da Destilaria Miriri, município de Capim, PB. O arranjo experimental foi um fatorial 2 x 4 (com e sem irrigação, e quatro doses de adubação de cobertura de $\mathrm{Ne} \mathrm{K}_{2} \mathrm{O}$ ) com três repetições. A lâmina de irrigação usada, $27,5 \mathrm{~mm}$, foi aplicada em intervalos de irrigação de 12 dias. As adubações de cobertura foram compostas dos elementos $\mathrm{Ne} \mathrm{K}_{2} \mathrm{O}$ aplicando-se respectivamente: $\mathrm{AC}_{1}=44$ e 41, $\mathrm{AC}_{2}=86$ e 81, $\mathrm{AC}_{3}=157$ e 148; e $\mathrm{AC}_{4}=236$ e $222 \mathrm{~kg} \mathrm{ha}^{-1}$. As interações estudadas regime de irrigação x doses de adubação de cobertura foram não-significativas. Quando submetida à irrigação, a cultura apresentou melhores rendimentos em todas as variáveis avaliadas, exceto peso médio do colmo, quando comparada a não irrigada. Nas parcelas irrigadas, a dose de adubação influenciou significativamente, segundo um comportamento linear positivo, os rendimentos de colmos, açúcar e álcool, enquanto que nas parcelas não irrigadas esse efeito só foi evidenciado no rendimento dos colmos. A máxima dose de adubação de cobertura utilizada no presente trabalho não foi suficiente para se atingir picos máximos de rendimentos, seja no regime irrigado ou não.
\end{abstract}

Termos para indexação: Cana-de-açúcar, adubação, irrigação, açúcar, álcool.

\begin{abstract}
This research had the objective of evaluating the effect of different manuring levels of $\mathrm{N}$ and $\mathrm{K}_{2} \mathrm{O}$ on the growth and yield of the sugar-cane (Saccharum spp, cultivate SP79 1011) first leaf, cultivated with and without irrigation. The experiment was conducted in the farm of the Miriri distillery, in the municipality of Capim, Paraíba state. The experimental scheme had a factorial type $2 \times 4$ (with and without irrigation and four levels of manuring of $\mathrm{N}_{\text {and }} \mathrm{K}_{2} \mathrm{O}$ ) with three replications. The water irrigation depth used, $27.5 \mathrm{~mm}$, was applied in intervals of 12 days. The manurings were composed of the elements $\mathrm{N}$ and $\mathrm{K}_{2} \mathrm{O}$, in levels based on recommendations of the consultants of the Miriri distillery, equal to $\mathrm{AC}_{1}=44 \mathrm{~kg} \mathrm{ha}^{-1}$ of nitrogen and $41 \mathrm{~kg} \mathrm{ha}^{-1}$ of potassium, $\mathrm{AC}=86 \mathrm{~kg} \mathrm{ha}{ }^{-1}$ of nitrogen and $81 \mathrm{~kg} \mathrm{ha}^{-1}$ of potassium, $\mathrm{AC}_{3}=157 \mathrm{~kg} \mathrm{ha}^{-1}$ of nitrogen and $148 \mathrm{~kg} \mathrm{ha}^{-1}$ of potassium, and $\mathrm{AC}_{4}=236 \mathrm{~kg} \mathrm{ha}^{-1}$ of nitrogen and $222 \mathrm{~kg} \mathrm{ha}^{-1}$ of potassium. When submitted to irrigation, the crop presented better yield in all appraised variables. In the irrigated parcels, the manuring affected significantly, through a positive linear behavior, the production of stems, sugar and alcohol, while in the non-irrigated parcels this effect was shown only for the production of stems. The maximum level of manurring in this work was not enough to get the maximum yield colm peak, using irrigation management or not.
\end{abstract}

Index terms: Sugarcane, manuring, irrigation, sugar, alcohol.

(Recebido para publicação em 21 julho de 2004 e aprovado em 30 de março de 2005)

\section{INTRODUÇÃO}

O Brasil é o maior produtor mundial de cana-deaçúcar, segundo o IBGE (2001), com área cultivada de 5.022 .490 ha e um rendimento médio de $69.445 \mathrm{~kg} \mathrm{ha}^{-1} \mathrm{de}$ colmos, gerando uma receita anual de 6,68 bilhões de reais. Esta cultura é importante, seja no aspecto social como no econômico, pois da sua industrialização são obtidos o açúcar e o álcool, sendo o açúcar um dos principais produtos brasileiros, e o seu cultivo é uma das maiores fontes de emprego por unidade de superfície, comparado com outras atividades agrícolas.

A Paraíba ocupa a sexta posição no cenário nacional, produzindo 8,9 milhões de toneladas, o que the confere uma receita anual de 120 milhões de reais, fazendo com que a cana-de-açúcar seja o produto agropecuário mais importante do Estado. No entanto, a produtividade da canade-açúcar na Paraíba é a mais baixa entre os maiores Estados produtores, 41,2 tha-1 (IBGE, 2002). Os principais fatores da baixa produtividade no Estado são o empobrecimento do solo e a baixa pluviosidade, os quais apontam para uma política de pesquisa com adubação e irrigação, visando resultados diretos, como aumento da produtividade e dos rendimentos de açúcar e álcool e, como efeito indireto diminuição da área plantada, minimizando custos de transporte, plantio e tratos culturais, além de liberar área para diversificação e/ou rotação de culturas e preservação do meio ambiente.

${ }_{1}^{1}$ Agrônoma pela CCA/UFPB - Rua Pedro Magalhães de Moura, 16 - 58960-000 - Bonito de Santa Fé, PB.

${ }^{2}$ Doutorando em Recursos Naturais - CCT/UFCG - pesquisador da usina Miriri - Capim, PB.

${ }^{3}$ Professores DEAg/CCT/UFCG - Av. Aprígio Veloso, 882 - Bodocongó, Bloco CM - 58109-970 - Campina Grande, PB - zedantas@deag.ufcg.edu.br

${ }^{4}$ Doutorando em Recursos Naturais - CCT/UFCG, Professor ESAM, Mosoró, RN - rpordeus@esam.br 
Doorenbos \& Kassan (1994) relatam que os rendimentos da cana-de-açúcar produzida em condições de sequeiro nos trópicos úmidos, variam entre 70 a $100 \mathrm{t} \mathrm{ha}^{-1} \mathrm{e}$, nos trópicos e sub-trópicos secos, com irrigação, rendimentos entre 100 e $150 \mathrm{t} \mathrm{ha}^{-1}$, que podem ser considerados satisfatórios. Os autores comentam, ainda, que o teor de açúcar parece diminuir ligeiramente com o aumento do rendimento de colmos da cultura e, para isto, deve-se evitar o crescimento exuberante durante a maturação da cana, o que pode ser obtido com temperaturas baixas, dose reduzida de nitrogênio e suprimento limitado de água.

A cana-de-açúcar é uma planta do grupo $\mathrm{C}_{4}$, as quais possuem maior eficiência fotossintética devido, provavelmente, à compartimentação de enzimas e às características anatômicas das folhas, tendo como consequiência taxa de fotorrespiração baixa ou ausente (MACHADO, 1987). Ainda segundo este autor, a produtividade da cana-de-açúcar depende da eficiência da integração do seu sistema produtivo formado pelas folhas fotossinteticamente ativas, do escoamento e distribuição do produto fotossintetizado, do consumo pela planta no seu desenvolvimento e reprodução e do acúmulo e armazenamento de sacarose.

Azeredo (1997) estudou o efeito de quatro doses de nitrogênio $\left(0,60,120\right.$ e $\left.180 \mathrm{~kg} \mathrm{ha}^{-1}\right)$ em cana-de-açúcar, em dois solos do Estado do Rio de Janeiro. A aplicação de nitrogênio resultou em resposta diferenciada sobre o desenvolvimento e produção final de colmos e de açúcar na cana-planta, mas não foram suficientes para afetar os teores de pol, fibras e açúcares totais da cana, sendo as variações nas produções de açúcar ha ${ }^{-1}$ resultantes das produções de colmos ha' ${ }^{-1}$.

Azevedo (2002), em experimento de campo realizado na Destilaria Miriri, no Estado da Paraíba, com a cultivar de cana-de-açúcar SP791011, cana-planta, em doses de adubação de cobertura de $85 \mathrm{~kg} \mathrm{ha}^{-1}$ ( $44 \mathrm{~kg}$ de N mais $41 \mathrm{~kg}$ de $\mathrm{K}_{2} \mathrm{O}$ ), $167 \mathrm{~kg} \mathrm{ha}^{-1}$ ( $86 \mathrm{~kg}$ de $\mathrm{N}$ mais $81 \mathrm{~kg}$ de $\left.\mathrm{K}_{2} \mathrm{O}\right), 305 \mathrm{~kg} \mathrm{ha}^{-1}$ ( $157 \mathrm{~kg}$ de N mais $148 \mathrm{~kg}$ de $\mathrm{K}_{2} \mathrm{O}$ ) e 458 $\mathrm{kg} \mathrm{ha}^{-1}$ ( $236 \mathrm{~kg}$ de $\mathrm{N}$ mais $222 \mathrm{~kg}$ de $\mathrm{K}_{2} \mathrm{O}$ ) e aplicando lâmina de irrigação de $27,5 \mathrm{~mm}$, encontrou produtividade média de colmos de 82,78, 86,20, 92,78 e 95,93 $\mathrm{t} \mathrm{ha}^{-1}$, o que proporcionou rendimentos médios de açúcar de 13,65, 13,76, 15,61 e 15,33 t ha ${ }^{-1}$ e rendimentos médios de álcool de $9570,9664,10983$ e $115301 \mathrm{ha}^{-1}$.

Matioli et al. (1998) empregando uma função que relaciona produção com consumo de água, na região de Ribeirão Preto, SP, durante o primeiro estágio de desenvolvimento da cultura (estabelecimento mais período vegetativo inicial), com lâmina mensal de até $30 \mathrm{~mm}$ verificaram que a irrigação complementar proporcionou aumento de produtividade em até $30,4 \mathrm{tha}^{-1}$ para as socas de início, até meados de safra (maio a julho), enquanto para as socas de fim de safra (setembro a novembro), o aumento de produtividade foi bastante reduzido, em torno de 3,2 a 8,4 tha $^{-1}$.

Tanto a cana-planta como as soqueiras apresentam boa resposta a potássio. O excesso de potássio no solo e/ ou sua falta, pode diminuir a qualidade da matéria-prima, influenciando as porcentagens do teor de sacarose (Pol) e a fibra industrial da cana. Santos et al. (1979) afirmam que o potássio provocou aumento na produtividade com adubação de até $440 \mathrm{~kg}_{\text {de }} \mathrm{K}_{2} \mathrm{O} \mathrm{ha}^{-1}$ e aumentou o teor de açúcar teórico recuperável nos experimentos de campo, em algumas regiões do Nordeste.

Haag et al. (1987) pesquisando relatórios do PLANALSUCAR, estação experimental de Alagoas, no período de 1967 a 1974, analisou a influência dos nutrientes N, P, K nas qualidades tecnológicas da cana-de-açúcar e concluíram que, em alguns casos, o nitrogênio quando aplicado em quantidades inferiores a $50 \mathrm{~kg} \mathrm{ha}^{-1}$ causou efeito depressivo; até $120 \mathrm{~kg} \mathrm{ha}^{-1}$, aumentou o pol e diminuiu a quantidade de açúcares redutores e, a partir daí, provocou efeitos contrários em ambas as características. Muchow et al. (1996) afirmam que um fator que contribui para o baixo rendimento da cultura, em termos de qualidade, é o manejo equivocado da adubação nitrogenada. Os autores trabalhando com doses de $\mathrm{N}$ de 56, 107 e $268 \mathrm{~kg} \mathrm{ha}^{-1}$, encontraram valores de PCC de 16,1, 14,9 e $13,1 \%$, respectivamente.

O Nordeste brasileiro vem sofrendo com as baixas precipitações ocorridas na região nos últimos anos, que ocasionaram queda na produção de cana-de-açúcar, levando os produtores a recorrerem a novas técnicas, como a irrigação suplementar, para a melhoria do cultivo. No entanto para uma melhor eficiência do uso da água de irrigação é importante a utilização de fertilização equilibrada, de maneira eficiente e lucrativa. Dentro desta perspectiva, o presente trabalho teve como objetivo avaliar o efeito de diferentes doses de $\mathrm{N} \mathrm{e}$ $\mathrm{K} 2 \mathrm{O}$, aplicados em cobertura, no desenvolvimento, rendimento e na qualidade da cana-soca (Saccharum spp, cultivar SP79 1011) cultivada com e sem irrigação.

\section{MATERIALEMÉTODOS}

O trabalho foi desenvolvido na fazenda Capim, de propriedade da Destilaria Miriri, município de Capim, PB,

Ciênc. agrotec., Lavras, v. 29, n. 4, p. 753-760, jul./ago., 2005 
situada na latitude de $6^{0} 56^{\prime} \mathrm{S}$, na longitude de $35^{\circ} 07^{\prime} \mathrm{W}$, durante o período de setembro de 2001 a setembro de 2002. O solo predominante na área experimental é o Argissolo Vermelho Amarelo (EMBRAPA, 1999), cujas características químicas e físicas se encontram na (Tabela 1). A irrigação foi suplementar e realizada no período de 10 outubro de 2001 a 31 de maio de 2002, ocorrendo a partir dessa data as precipitações normais na região. A lâmina de irrigação utilizada de $27,5 \mathrm{~mm}$ foi calculada com base na evaporação diária do tanque Classe A, utilizando-se coeficientes de tanque, Kp, (DOORENBOS \& PRUITT, 1997) e de cultura, Kc, (DOORENBOS \& KASSAN, 1994) definidos em função das condições locais e foi aplicada por um sistema de pivô central rebocável em intervalos de 12 dias.

$\mathrm{O}$ arranjo experimental foi fatorial 2 x 4 (com e sem irrigação e quatro doses de adubação de cobertura AC de N e $\mathrm{K}_{2} 0$ ) com três repetições, dispostas em blocos ao acaso, sendo: $\mathrm{AC}_{1}-44 \mathrm{~kg}$ de $\mathrm{N}$ mais $41 \mathrm{~kg}$ de $\mathrm{K}_{2} \mathrm{O} \mathrm{ha}^{-1}, \mathrm{AC}_{2}-86 \mathrm{~kg}$ de $\mathrm{N}$ mais $81 \mathrm{~kg}$ de $\mathrm{K}_{2} \mathrm{O} \mathrm{ha}^{-1}, \mathrm{AC}_{3}-157 \mathrm{~kg}$ de $\mathrm{N}$ mais 148 $\mathrm{kg}$ de $\mathrm{K}_{2} \mathrm{O} \mathrm{ha}^{-1} \mathrm{e} \mathrm{AC}_{4}-236 \mathrm{~kg}$ de $\mathrm{N}$ mais $222 \mathrm{~kg} \mathrm{de}_{2} \mathrm{O} \mathrm{ha}^{-1}$. As parcelas foram constituídas de 5 fileiras de plantas de $12 \mathrm{~m}$ de comprimento, espaçadas $1,2 \mathrm{~m}$ com uma área total de $72 \mathrm{~m}^{2}$. A área útil da parcela foi de $36 \mathrm{~m}^{2}$, compreendendo as três fileiras centrais com $10 \mathrm{~m}$ de comprimento constituindo-se a bordadura de uma fileira de cana de cada lado e 1,0 m em cada extremidade da parcela. O plantio da cana-planta ocorreu em agosto de 2000, com colmos inteiros, deitados no interior do sulco, na densidade de 18 gemas por metro. O solo foi preparado com grade de disco aradora e sulcamento a uma profundidade de $0,3 \mathrm{~m}$ e espaçamento de $1,2 \mathrm{~m}$, aplicando-se de forma mecanizada, gesso na proporção de $1000 \mathrm{~kg} \mathrm{ha}^{-1}$ e adubação de fundação na quantidade de $100 \mathrm{~kg} \mathrm{ha}^{-1}$ da fórmula 00-18-00. O corte da cana-planta foi feito em de setembro de 2001. Os tratos culturais dispensados à cana-soca foram a irrigação, a aplicação de herbicidas, as adubações de cobertura com $\mathrm{N}_{\text {e }} \mathrm{K} \mathrm{O}$ e colheita doze meses depois do corte da cana-planta.

As adubações de cobertura com $\mathrm{Ne} \mathrm{K}_{2} \mathrm{O}$, aplicadas em linhas, foram fracionadas e realizadas em quatro momentos: no dia 02/01/2002 foi aplicado, $44 \mathrm{~kg} \mathrm{ha}^{-1} \mathrm{de} \mathrm{N}$ mais $41 \mathrm{~kg} \mathrm{ha}^{-1}$ de $\mathrm{K}_{2} \mathrm{O}$ em todos os tratamentos; no dia 02/ 04/2002 foi aplicado $42 \mathrm{~kg} \mathrm{ha}^{-1}$ de $\mathrm{N}$ mais $40 \mathrm{~kg} \mathrm{ha}^{-1}$ de $\mathrm{K}_{2} \mathrm{O}$ nos tratamentos $\mathrm{AC}_{2}, \mathrm{AC}_{3}$ e $\mathrm{AC}_{4}$; no dia 03/05/2002 foi aplicado $71 \mathrm{~kg} \mathrm{ha}^{-1}$ de $\mathrm{N}$ mais $67 \mathrm{~kg} \mathrm{ha}^{-1}$ de $\mathrm{K}_{2} \mathrm{O}$ nos tratamentos $\mathrm{AC}_{3}$ e $\mathrm{AC}_{4}$ e no 03/06/2002 foi aplicado $79 \mathrm{~kg} \mathrm{ha}^{-1} \mathrm{de} \mathrm{N}$ mais $74 \mathrm{~kg} \mathrm{ha}^{-1}$ de $\mathrm{K}_{2} \mathrm{O}$ no tratamento $\mathrm{AC}_{4}$. A proporção entre os nutrientes $\mathrm{N} \mathrm{e}_{2} \mathrm{O}$ na adubação de cobertura foi de 1,0 para 0,94 .

TABELA 1 - Características químicas e físico-hídricas do solo da área experimental.

\begin{tabular}{|c|c|c|}
\hline Características & Unidade & Valor \\
\hline pH em água $(1: 25)$ & - & 4,54 \\
\hline $\mathrm{P}$ & $\mathrm{mg} \mathrm{dm}^{-3}$ & 26,4 \\
\hline $\mathrm{K}$ & $\mathrm{mg} \mathrm{dm}^{-3}$ & 0,5 \\
\hline $\mathrm{Ca}$ & $\mathrm{mmol}_{\mathrm{c}} \mathrm{dm}^{-3}$ & 10,9 \\
\hline $\mathrm{Mg}$ & $\mathrm{mmol}_{\mathrm{c}} \mathrm{dm}^{-3}$ & 6,3 \\
\hline $\mathrm{Al}$ & $\mathrm{mmol}_{\mathrm{c}} \mathrm{dm}^{-3}$ & 7,2 \\
\hline S & $\mathrm{mmol}_{\mathrm{c}} \mathrm{dm}^{-3}$ & 0,7 \\
\hline MO & $\mathrm{g} \mathrm{dm}^{-3}$ & 8,0 \\
\hline Areia & $\mathrm{g} \mathrm{kg}^{-1}$ & 670,0 \\
\hline Silte & $\mathrm{g} \mathrm{kg}^{-1}$ & 23.0 \\
\hline Argila & $\mathrm{g} \mathrm{kg}^{-1}$ & 307 \\
\hline Densidade & $\mathrm{kg} \mathrm{m} \mathrm{m}^{-3}$ & 1,39 \\
\hline Capacidade de campo- $\Theta_{\mathrm{CC}}$ & $\mathrm{cm}^{3} \mathrm{~cm}^{-3}$ & 0,126 \\
\hline Ponto de murchamento permanente- $\theta$ pMP & $\mathrm{cm}^{3} \mathrm{~cm}^{-3}$ & 0.061 \\
\hline
\end{tabular}


As quantidades dos elementos $\mathrm{N}$ e $\mathrm{K}_{2} \mathrm{O}$ foram definidas, tomando-se como ponto de partida as dosagens recomendadas pela equipe de consultores da Destilaria Miriri ( $86 \mathrm{~kg}$ de $\mathrm{N}$ mais $81 \mathrm{~kg}$ de $\mathrm{K}_{2} \mathrm{O} \mathrm{ha}^{-1}$ ), tanto para a cana-planta como para a primeira soca, que se baseia em análise do solo e no rendimento econômico da cultura sob condições de sequeiro. As fontes de nitrogênio e potássio foram uréia e cloreto de potássio, respectivamente.

A área útil da parcela foi colhida contando-se o número de colmos, pesando-se e calculando-se a produção de colmos em kg ha-1. Também, foi cortada ao acaso uma touceira de cana-de-açúcar (na qual estão representados colmos com diferentes diâmetros) que foi analisada no laboratório da destilaria, onde foram determinadas as características tecnológicas da cana-de-açúcar, de acordo com Caldas (1998): sólidos solúveis ('Brix em \%), sacarose (Pol do caldo em \%) e porcentagem bruta de açúcar (PCC).

Os rendimentos brutos de açúcar e de álcool foram calculados de acordo com Caldas (1998), utilizando-se as seguintes fórmulas:

\section{Rendimento Bruto de Açúcar}

$$
\mathrm{R}_{\mathrm{AÇUCAR}}=(\mathrm{PCCXPC}) \times 0,01
$$

Em que: $\mathrm{R}_{\mathrm{ACCúCAR}}$-rendimento de açúcar em tha ${ }^{-1} ; \mathrm{PCC}$ - quantidade de açúcar em \% contido nos colmos e determinada em laboratório; PC - produção de colmos em t ha ${ }^{-1}$.

\section{Rendimento Bruto de Álcool}

$$
\mathrm{R}_{\text {ALCoOL }}=(((\mathrm{PCCxF})+\mathrm{ARL}) \times F g) \times P C x 0,01
$$

Em que: $\mathrm{R}_{\text {ÁLCOOL }}$ - rendimento de álcool bruto em $\mathrm{m}^{3} \mathrm{ha}^{-1} ; \mathrm{F}$ - fator de transformação estequiométrica de sacarose em uma molécula de glicose mais uma de frutose, igual a 1,052; ARL - açúcares redutores livres em \%; Fg fator de Gay Lussac igual a 0,6475.

\section{RESULTADOS E DISCUSSÃO}

\section{a) Características organográficas}

Após análise de variância realizada com os dados obtidos, comprovou-se que com exceção do peso dos colmos todas as demais variáveis organográficas estudadas responderam significativamente à irrigação, enquanto que apenas o número e o comprimento dos colmos responderam significativamente à adubação de cobertura. Nenhuma das variáveis analisadas apresentou interação nível de adubação de cobertura versus regime de irrigação significativa.

Os valores referentes às médias de número de colmos (NC), comprimento de colmos (CC), diâmetro de colmos (DC), número de internódios (NI) e peso do colmo (PC), observados em função dos regimes de irrigação e doses de adubação de cobertura (AC), são apresentados na Tabela 2.

TABELA 2 - Médias para número de colmos (NC), comprimento de colmos (CC), diâmetro de colmos (DC), número de internódios (NI) e peso do colmo (PC) em função dos regimes de irrigação e doses de adubação de

\begin{tabular}{|c|c|c|c|c|c|}
\hline Dose de adubação & $\begin{array}{c}\text { NC } \\
\left(\mathbf{n}^{\mathbf{0}} \mathbf{h a}^{-1}\right) \\
\end{array}$ & $\begin{array}{l}\mathrm{CC} \\
(\mathbf{c m})\end{array}$ & $\begin{array}{c}\text { DC } \\
(\mathbf{m m})\end{array}$ & $\begin{array}{c}\text { NI } \\
\left(n^{0} \operatorname{colmos}^{-1}\right)\end{array}$ & $\begin{array}{r}\text { PC } \\
(\mathrm{g})\end{array}$ \\
\hline \multicolumn{6}{|c|}{ Com Irrigação } \\
\hline 85 & 73981 & 217,33 & 23,60 & 22 & 913,33 \\
\hline 167 & 85370 & 234,00 & 22,47 & 23 & 1028,33 \\
\hline 305 & 91204 & 250,33 & 25,27 & 23 & 1143,33 \\
\hline 458 & 93518 & 237,67 & 24,30 & 23 & 1153,33 \\
\hline Média & 86019 A & $234,83 \mathrm{~A}$ & $23,91 \mathrm{~A}$ & $23 \mathrm{~A}$ & $1059,58 \mathrm{~A}$ \\
\hline $\mathrm{CV}(\%)$ & 7,72 & 6,19 & 6,58 & 5,07 & 9,26 \\
\hline \multicolumn{6}{|c|}{ Sem Irrigação } \\
\hline 85 & 67315 & 170,67 & 19,03 & 17 & 980,00 \\
\hline 167 & 78889 & 184,67 & 21,90 & 18 & 1000,00 \\
\hline 305 & 79722 & 197,00 & 22,20 & 18 & 1040,00 \\
\hline 458 & 87037 & 198,33 & 20,07 & 18 & 956,67 \\
\hline Média & 78241 B & $187,92 \mathrm{~B}$ & $20,80 \mathrm{~B}$ & $18 \mathrm{~B}$ & $994,17 \mathrm{~A}$ \\
\hline $\mathrm{CV}(\%)$ & 5,27 & 3,76 & 6,99 & 4,27 & 8,85 \\
\hline
\end{tabular}
cobertura (AC).

"Médias seguidas pela mesma letra, na coluna, não diferem estatisticamente pelo teste de tukey, a nível de 5\% de probabilidade.

Ciênc. agrotec., Lavras, v. 29, n. 4, p. 753-760, jul./ago., 2005 
Observou-se que o regime de irrigação aumentou o número de colmos com a média subindo de 78241 para 86019 colmos ha ${ }^{-1}$, cujos valores diferem estatisticamente pelo teste Tukey $(\mathrm{p}<0,05)$. Mesmo com irrigação, o valor obtido é inferior aos 90000 colmos ha-1 que, segundo Taupier \& Rodrigues (1999) são necessários para se atingir produtividades máximas. Azevedo (2002), em trabalho desenvolvido nesta mesma área, encontrou uma média geral de 81835 colmos ha ${ }^{-1}$ na cana-planta.

O regime irrigado $(234,83 \mathrm{~cm})$ proporcionou aumento de $25 \%$ no comprimento dos colmos em relação a regime sem irrigação $(187,92 \mathrm{~cm})$. Os comprimentos de colmos encontrados em ambos os regimes estão coerentes com os valores de literatura. Marciel et al. (2002) trabalhando com cana-soca, em solo de cerrado, no município de Pompeu, MG, avaliando a resposta da cultura à irrigação suplementar quando plantada no período de maio a agosto, verificaram comprimento médio de 102,2 cm sem irrigação e 253,7 cm com irrigação.

O regime irrigado possibilitou aumento de 13 e $22 \%$ no diâmetro e número de internódios do colmo, quando comparado à ausência de irrigação. Azevedo (2002) obteve resultados semelhantes aos encontrados com valores de diâmetro médio de 23,45 mm e 23 internódios por colmo para cana-planta.

$\mathrm{O}$ peso médio do colmo encontrado no presente trabalho, 1059,58 g e 994,17 g, com e sem irrigação, respectivamente, estão coerentes com os resultados de pesquisa da literatura. Azevedo (2002), em cana-planta, encontrou peso médio por colmo de $917,00 \mathrm{~g}$, inferior ao obtido nesta pesquisa. Silva (2002), estudando a resposta da cana-de-açúcar irrigada sob diferentes doses de adubação, também no mesmo local, encontrou um peso médio por colmo de $1384,17 \mathrm{~g}$, trabalhando com a variedade SP-716949.

A análise de regressão, para o fator quantitativo nível de adubação de cobertura, mostrou que com irrigação apenas as variáveis número e peso de colmos apresentaram comportamento linear positivo segundo as equações descritas na Tabela 3. Nas parcelas irrigadas, o maior número de colmos (95895) e o maior peso (1191 g) foram obtidos com o nível de adubação de cobertura correspondente a $458 \mathrm{~kg} \mathrm{ha}^{-1}$. Na ausência da irrigação as variáveis número e comprimento de colmos, apresentaram comportamento linear positivo, ou seja, quanto maior o nível de adubação de cobertura maior os valores dessas variáveis. A variável diâmetro de colmos apresentou comportamento quadrático, com o maior valor $(22,9 \mathrm{~mm})$ obtido com $299 \mathrm{~kg} \mathrm{ha}^{-1}$.

\section{b) Rendimento e qualidade da cana-de-açúcar}

Após análise de variância realizada com os dados obtidos, comprovou-se que todas as variáveis estudadas responderam significativamente ao efeito regime de irrigação e doses de adubação de cobertura, com exceção do PCC que não respondeu significativamente ao efeito da adubação de cobertura. Nenhuma das variáveis analisadas apresentou interação dose de adubação de cobertura versus regime de irrigação significativa.

Os valores referentes às médias de rendimento de colmos $\left(\mathrm{R}_{\text {colmos }}\right)$, parâmetros tecnológicos ( ${ }^{\circ}$ Brix, Pol, PCC), rendimento bruto do açúcar $\left(\mathrm{RB}_{\text {acúcar }}\right)$ e de álcool $\left(\mathrm{RB}_{\text {álcoo }}\right)$, observados em função dos regimes de irrigação e doses de adubação de cobertura (AC), utilizados são apresentados na Tabela 4 .

TABELA 3 - Equações de regressão para número de colmos (NC), peso do colmo (PC), comprimento de colmos (CC) e diâmetro de colmos (DC), em função da irrigação e da adubação de cobertura (AC).

\begin{tabular}{ccc}
\hline Regimes de irrigação & Equações de regressão & $\mathbf{R}^{2}$ \\
\hline Com Irrigação & $\mathrm{NC}=73,7486+0,0483^{* *} \mathrm{AC}^{1}$ & 0,82 \\
& $\mathrm{PC}=896,8691+0,6412^{*} \mathrm{AC}$ & 0,84 \\
& $\mathrm{NC}=66,6815+0,0455^{* *} \mathrm{AC}$ & 0,84 \\
Sem Irrigação & $\mathrm{CC}=169,4816+0,0726^{* *} \mathrm{AC}$ & 0,82 \\
& $\mathrm{DC}=15,8046+0,0478 \mathrm{AC}-0,00008^{*} \mathrm{AC}^{2}$ & 0,94 \\
\hline
\end{tabular}

${ }^{1} \mathrm{AC}$ - Adubação de cobertura, $\mathrm{kg} ;{ }^{*}$ e ${ }^{* *}$ significativo a nível de $5 \%$ e $1 \%$ de probabilidade pelo teste $\mathrm{F}$. 
TABELA 4 - Médias de rendimento médio de colmos $\left(\mathrm{R}_{\text {colmos }}\right)$, ${ }^{\circ}$ Brix, Pol, PCC, rendimento bruto de açúcar $\left(\mathrm{RB}_{\text {açúcar }}\right)$ e de álcool ( $\left.\mathrm{RB}_{\text {álcool }}\right)$ em função dos regimes de irrigação e doses de adubação de cobertura $(\mathrm{AC})$.

\begin{tabular}{|c|c|c|c|c|c|c|}
\hline $\begin{array}{c}\text { Dose de } \\
\text { adubação }\end{array}$ & $\begin{array}{l}R_{\text {colmos }} \\
\left(\mathbf{t ~ h a}^{-1}\right)\end{array}$ & $\begin{array}{l}{ }^{0} \text { Brix } \\
(\%)\end{array}$ & $\begin{array}{l}\text { Pol } \\
(\%)\end{array}$ & $\begin{array}{c}\text { PCC } \\
(\%)\end{array}$ & $\begin{array}{c}\mathbf{R B}_{\text {açúcar }} \\
\left(\mathrm{t} \mathrm{ha}^{-1}\right)\end{array}$ & $\begin{array}{l}\text { RB } \\
\left(\mathrm{m}^{3} \mathrm{ha}^{-1}\right)\end{array}$ \\
\hline \multicolumn{7}{|c|}{ Com Irrigação } \\
\hline 85 & 68,11 & 19,57 & 17,65 & 14,21 & 9,79 & 6,88 \\
\hline 167 & 89,89 & 19,63 & 17,37 & 14,04 & 12,32 & 8,89 \\
\hline 305 & 104,23 & 20,10 & 17,96 & 14,51 & 15.17 & 10,60 \\
\hline 458 & 107,69 & 19,73 & 17,60 & 14,36 & 15,48 & 10,83 \\
\hline Média & $92,48 \mathrm{~A}$ & $19,76 \mathrm{~A}$ & $17,65 \mathrm{~A}$ & $14,28 \mathrm{~A}$ & $13,18 \mathrm{~A}$ & $9,30 \mathrm{~A}$ \\
\hline $\mathrm{CV}(\%)$ & 11,30 & 3,38 & 4,27 & 4,31 & 12,63 & 12,62 \\
\hline \multicolumn{7}{|c|}{ Sem Irrigação } \\
\hline 85 & 64,69 & 18,70 & 15,96 & 13,13 & 8,49 & 6,00 \\
\hline 167 & 78,16 & 18,30 & 15,68 & 12,72 & 10,24 & 7,25 \\
\hline 305 & 82,34 & 18,83 & 15,86 & 13,04 & 10,76 & 7,55 \\
\hline 458 & 83,01 & 18,70 & 16,21 & 13,45 & 11,19 & 7,83 \\
\hline Média & $77,05 \mathrm{~B}$ & $18,63 \mathrm{~B}$ & $15,93 \mathrm{~B}$ & $13,08 \mathrm{~B}$ & $10,17 \mathrm{~B}$ & $7,16 \mathrm{~B}$ \\
\hline $\mathrm{CV}(\%)$ & 7,36 & 6,30 & 8,27 & 8,89 & 14,13 & 13,30 \\
\hline
\end{tabular}

"Médias seguidas pela mesma letra, na coluna, não diferem estatisticamente pelo teste de tukey, a nível de 5\% de probabilidade.

Observou-se que o regime de irrigação aumentou o $\mathrm{R}_{\text {colmos }}$ com a média subindo de 77,05 para 92,48 $\mathrm{tha}^{-1}$, cujos valores diferem estatisticamente pelo teste Tukey $(\mathrm{p}<0,05)$. O estresse hídrico foi a causa da diminuição do rendimento da cultura. Farias (2001) encontrou diferença de $28,26 \mathrm{t} \mathrm{ha}^{-1}$ entre a cana irrigada e a cana de sequeiro. Matioli et al. (1998), aplicando uma lâmina mensal de $30 \mathrm{~mm}$ verificaram que a irrigação complementar proporcionou aumento de produtividade em até $30,4 \mathrm{t} \mathrm{ha}^{-1}$ para cana soca.

Mesmo sem irrigação o valor obtido $\left(64,69 \mathrm{t} \mathrm{ha}^{-1}\right)$ na menor dose de adubação de cobertura ( $44 \mathrm{~kg}$ de $\mathrm{Ne} 41 \mathrm{~kg}$ de $\mathrm{K}_{2} \mathrm{O}_{\text {ha-1 }}$ ) é superior a média da Paraíba (IBGE, 2002). O maior valor da área irrigada $\left(107,69 \mathrm{t} \mathrm{ha}^{-1}\right)$ foi obtido com a maior dose de adubação e está coerente com recomendações de Doorenbos \& Kassan (1994) que consideram como bons, em áreas irrigadas, rendimentos acima de $100 \mathrm{tha}^{-1}$. Azevedo (2002) trabalhando com a mesma variedade, a mesma lâmina de irrigação e com a cana-planta encontrou produtividade média de $89,42 \mathrm{tha}^{-1}$.

A irrigação proporcionou aumentos significativos nas médias dos ${ }^{\circ}$ Brix, Pol, PCC, $\mathrm{RB}_{\text {aćúcar }}$ e $\mathrm{RB}_{\text {álcool }}$. Para o rendimento de açúcar houve um aumento de 22,8 enquanto que para o rendimento do álcool esse aumento foi de $23 \%$. O rendimento médio, para áreas irrigadas, $13,18 \mathrm{t} \mathrm{ha}^{-1}$ pode ser considerado bom. Wiedenfeld (1995) trabalhando com três condições de irrigação $(95,85$ e $65 \%$ da fração de esgotamento do solo) obteve rendimentos de 13; 10 e $8 \mathrm{t} \mathrm{ha}^{-1}$ de açúcar, respectivamente.

Mediante análise de regressão, as variáveis $\left(\mathrm{RB}_{\text {acúcar }}\right)$ e $\left(\mathrm{RB}_{\text {alcool }}\right)$ apresentaram comportamento linear positivo apenas para o regime irrigado. Enquanto que a variável $\left(\mathrm{R}_{\text {colmos }}\right)$ apresentou comportamento linear positivo tanto para a presença ou ausência de irrigação, segundo as equações descritas na Tabela 5. Nas parcelas irrigadas, o maior $\mathrm{R}_{\text {colmos }}\left(92,48 \mathrm{tha}^{-1}\right), \mathrm{R}_{\text {ą̧úcar }}\left(13,18 \mathrm{tha}^{-1}\right)$ e o maior $R_{\text {alcool }}\left(9,30 \mathrm{~m}^{3} \mathrm{ha}^{-1}\right)$ foram obtidos com a dose de adubação de cobertura correspondente a máxima adubação usada ( $458 \mathrm{~kg} \mathrm{ha}^{-1}$ ), evidenciando que doses de adubação de cobertura maiores podem ainda aumentar os rendimentos de colmos ( $\left.\mathrm{t} \mathrm{ha}{ }^{-1}\right)$ nas condições do presente trabalho. Observa-se que o rendimento de açúcar aumentou com o aumento da dose de adubação, em ambos os regimes estudados. Este fenômeno também foi observado por Azevedo (2002), quando trabalhou com cana-planta. 
TABELA 5 - Equações de regressão para rendimento de colmos $\left(\mathrm{R}_{\text {colmos }}\right)$, rendimento bruto do açúcar $\left(\mathrm{RB}_{\text {açúcar }}\right)$, e rendimento bruto do álcool ( $\left.\mathrm{RB}_{\text {álcool }}\right)$ em função dos regimes de irrigação e doses de adubação de cobertura (AC).

\begin{tabular}{ccc}
\hline Regimes de irrigação & Equações de regressão & $\mathbf{R}^{\mathbf{2}}$ \\
\hline Com Irrigação & $\mathrm{R}_{\text {colmos }}=67,06666+0,1001^{* *} \mathrm{AC}$ & 0,83 \\
& $\mathrm{RB}_{\text {açúcar }}=9,3362+0,0151^{* *} \mathrm{AC}$ & 0,86 \\
& $\mathrm{RB}_{\text {álcool }}=6,7019+0,0102^{* *} \mathrm{AC}$ & 0,84 \\
Sem Irrigação & $\mathrm{R}_{\text {colmos }}=66,0666+0,0433^{* *} \mathrm{AC}$ & 0,69 \\
\hline${ }^{1} \mathrm{AC}-$ Adubação de cobertura, $\mathrm{kg} ;{ }^{*}{ }^{*} \mathrm{e}^{* *}$ significativo a nível de 5\% e $1 \%$ de probabilidade pelo teste $\mathrm{F}$ &
\end{tabular}

${ }^{1} \mathrm{AC}$ - Adubação de cobertura, kg; ${ }^{*}$ e ${ }^{* *}$ significativo a nível de $5 \%$ e $1 \%$ de probabilidade pelo teste $\mathrm{F}$.

\section{CONCLUSÕES}

O regime de irrigação proporcionou melhor rendimento em todas as variáveis avaliadas, exceto no peso médio dos colmos, quando comparado ao não irrigado.

As interações estudadas, regimes de irrigação versus doses de adubação de cobertura, foram nãosignificativas para todas as variáveis estudadas, mostrando que os dois fatores atuam independente um do outro.

Nas parcelas irrigadas, a dose de adubação influenciou significativamente, segundo um comportamento linear positivo, os rendimento de colmos, açúcar e álcool, já nas parcelas não irrigadas, esse efeito só foi evidenciado no rendimento dos colmos.

A maior dose de adubação de cobertura utilizada ( $236 \mathrm{~kg}$ de $\mathrm{N}$ e $22 \mathrm{~kg}$ de $\mathrm{K}_{2} \mathrm{O} \mathrm{ha}^{-1}$ ) não foi suficiente para se atingir picos máximos de rendimento de colmos $\left(\mathrm{t} \mathrm{ha}^{-1}\right)$, seja no regime irrigado ou não.

\section{AGRADECIMENTOS}

Ao CNPq pelo apoio financeiro e a Destilaria Miriri, especialmente ao Diretor Superintendente Dr. Gilvan Celso Cavalcanti, ao Gerente Agrícola Dr. Gabriel Saturnino de Oliveira e ao Diretor Industrial Dr. Emanuel Pinheiro de Melo, pela área experimental e apoio nos trabalhos de campo.

\section{REFERÊNCIASBIBLIOGRÁFICAS}

AZEREDO, D. F. Eficiência da adubação nitrogenada em cana-de-açúcar em dois solos do estado do Rio de Janeiro: cana-planta. 1997. 167 f. Tese (Doutorado em Agronomia) - Universidade Federal do Rio de Janeiro, Campos, 1997.
AZEVEDO, H. M. de. Resposta da cana-de-açúcar a doses de irrigação e de adubação de cobertura nos tabuleiros costeiros da Paraíba. 2002. 112 f. Tese (Doutorado em Recursos Naturais) - Universidade Federal de Campina Grande, Campina Grande, 2002.

CALDAS, C. Manual de análises selecionadas para indústrias sucroalcooleiras. Maceió: Sindicato da Indústria e do Álcool do Estado de Alagoas, 1998. $424 \mathrm{p}$.

DOORENBOS, J.; KASSAM, A. H. Efeito da água no rendimento das culturas. Campina Grande: UFPB, 1994. 306 p. (FAO. Estudos de Irrigação e Drenagem, 33).

DOORENBOS, J.; PRUITT, W. O. Necessidades hídricas das culturas. Campina Grande: UFPB, 1997. 204 p. (FAO. Estudos de Irrigação e Drenagem, 24).

EMPRESA BRASILEIRA DE PESQUISA AGROPECUÁRIA. Centro Nacional de Pesquisa de Solo. Sistema brasileiro de classificação do solo. Brasília, 1999. $412 \mathrm{p}$.

FARIAS, C. H. A. Desenvolvimento morfofisiológico da cana de açúcar em regime irrigado e sequeiro na Zona da Mata Paraibana. 2001. 74 f. Dissertação (Mestrado em Engenharia Agrícola) - Universidade Federal de Campina Grande, Campina Grande, 2001.

HAAG, H. P.; DECHEN, A. R.; CARMELLO, Q. A. C. Nutrição mineral da cana-de-açúcar. In: PARANHOS, S. B. (Coord.). Cana-de-açúcar: cultivo e utilização. Campinas: Fundação Cargill, 1987. v. 1, p. 88-162. 
INSTITUTO BRASILEIRO DE GEOGRAFIA E ESTATÍSTICA. Censo Agropecuário 1995-1996.

Disponível em: <http//www.sidra.ibge.gov.br>. Acesso em: 10 maio 2002.

INSTITUTO BRASILEIRO DE GEOGRAFIA E ESTATÍSTICA. Produção agrícola municipal 2001. 2001. Disponível em: <http://www.ibge.gov.br>. Acesso em: 20 maio 2003.

MACHADO, E. C. Fisiologia da produção de cana-de-açúcar. In: PARANHOS, S. B. (Coord.). Cana-de-açúcar: cultivo e utilização. Campinas: Fundação Cargill, 1987. v. 1, p. 57-87.

MARCIEL, M. L. et al. Manejo da irrigação na cana-soca no cerrado de Minas Gerais. In: CONGRESSO NACIONAL DA SOCIEDADE DOS TÉCNICOS AÇUCAREIROS E ALCOOLEIROS DO BRASIL, 8., 2002, Recife. Anais... Recife: STAB, 2002.

MATIIOLI, C. S. et al. Aumento de produtividade da cultura da cana-de-açúcar sob irrigação complementar na região de Ribeirão Preto, SP. In: CONGRESSO BRASILEIRO DE ENGENHARIAAGRÍCOLA, 27., 1998, Poços de Caldas, MG. Anais... Lavras: UFLA/SBEA, 1998. v. 2, p. 16-18.
MUCHOW, R. C.; ROBERTSON, M. J.; WOOD, A. W. Effect of nitrogen on the time course of sucrose accumulation in sugar cane. Field Crops Research, Amsterdam, v. 7, p. 143-153, 1996.

SANTOS, M. A. C. dos; SOBRAL, A. F. de; CORDEIRO, D. A.; ARAÚJO, J. D. L. de. Adubação da cana-de-açúcar: resumo informativo. Carpina: IAA/PLANALSUCAR, 1979. 3 p.

SILVA, A. B. Resposta da cana-de-açúcar irrigada sob diferentes doses de adubação. 2002. 64 f. Dissertação (Mestrado em Engenharia Agrícola) - Universidade Federal de Campina Grande, Campina Grande, 2002.

TAUPIER, L. O. G.; RODRIGUES, G. G. A cana-deaçúcar. In: CIDCA. Manual dos derivados da cana-deaçúcar: diversificação, matérias primas, derivados do bagaço, derivados do melaço, outros derivados, resíduos, energia. Brasília: ABIPTI, 1999. v. 1, cap. 2, p. 21-27.

WIEDENFELD, R. P. Effects of irrigation and N fertilizer application on sugar cane yield and quality. Field Crop Research, Amsterdam, v. 43, p. 101-108, 1995. 